\title{
ESTRUCTURA, COMPOSICIÓN FLORÍSTICA Y DIVERSIDAD DEL MATORRAL ESPINOSO TAMAULIPECO, MÉXICO
}

\author{
STRUCTURE, FLORISTIC COMPOSITION AND DIVERSITY IN THE \\ TAMAULIPAN THORNSCRUB, MEXICO
}

\author{
Carlos Alberto Mora Donjuán ${ }^{1}$, Eduardo Alanís Rodríguez, Javier Jiménez Pérez, Marco Aurelio González \\ Tagle, José Israel Yerena Yamallel y Luis Gerardo Cuellar Rodríguez
}

\begin{abstract}
Resumen
La presente investigación tuvo la finalidad de evaluar la composición y la diversidad de las especies arbóreas y arbustivas de un área de referencia del matorral espinoso tamaulipeco (MET). Se establecieron cuatro sitios de muestreo de $40 \times 40 \mathrm{~m}$ en un área del MET, donde se realizó un censo de todos los individuos $\left(d_{0.10 \mathrm{~m}}>5 \mathrm{~cm}\right)$. Se registró información dasométrica de altura $(h)$, diámetro $\left(d_{0.10 \mathrm{~m}}\right)$ y cobertura de copa. Se determinaron las variables estructurales de abundancia $\left(A R_{i}\right)$, dominancia $\left(D R_{i}\right)$, frecuencia $\left(F R_{i}\right)$ e Índice de Valor de Importancia $(I V I)$, así como la estimación del índice de riqueza de Margalef $\left(D_{M g}\right)$ y el índice de diversidad de Shannon-Weaver $\left(H^{\prime}\right)$. Se registraron 21 especies, distribuidas en 20 géneros y 14 familias. La familia con mayor riqueza fue Fabaceae, con siete especies. La comunidad vegetal estudiada se integra principalmente de las especies Acacia amentacea, Diospyros texana y Havardia pallens, cuyos respectivos índices de valor de importancia fueron $24.94 \%, 15.57 \%$ y $13.01 \%$. Para la comunidad estudiada se registró un valor de $H^{\prime}$ de 1.94 y un valor de $D_{M g}$ de 2.0.

Palabras clave: riqueza de especies, índice de valor de importancia, índice de Margalef, índice de Shannon-Wiener, Nuevo León, variables estructurales.
\end{abstract}

\begin{abstract}
This research aimed to evaluate the structure, floristic composition and diversity of tree and shrub species of a reference community of Tamaulipan thornscrub (MET). We recorded the total number of trees $\left(d_{0.10 \mathrm{~m}}>5 \mathrm{~cm}\right)$ in four $40 \times 40 \mathrm{~m}$ sampling sites. Height $(h)$, diameter $\left(d_{0.10 \mathrm{~m}}\right)$ and canopy cover were recorded, and abundance $\left(A R_{i}\right)$, dominance $\left(D R_{i}\right)$, frequency $\left(F R_{i}\right)$, Importance Value Index $(I V I)$, Margalef index $\left(D_{M g}\right)$, Shannon-Weaverr index $\left(H^{\prime}\right)$ were calculated. There were a total 21 species, distributed in 20 genus and 14 families. Fabaceae was the most speciose family, with seven species. The most common species in the community were Acacia amentacea $(I V I=24.94 \%)$, Diospyros texana $(15.57 \%)$ and Havardia pallens $(13.01 \%)$. The studied community had a diversity index of $H^{\prime}=1.94$ and Margalef species richness of $D_{M g}=2.0$.
\end{abstract}

Key words: species richness, importance value index, Nuevo León, structural variables.

\section{Introducción.}

La estructura y la diversidad son, junto con la densidad, características importantes para la descripción cuantitativa de cualquier rodal de vegetación. La diversidad es un concepto que abarca diferentes interpretaciones, como la diversidad dimensional y estructural, aunque en su versión más simple se emplea como sinónimo de diversidad de especies. La estructura de una comunidad vegetal hace referencia, entre otras cosas, a la distribución de las principales características arbóreas en el espacio, teniendo especial importancia la distribución de las diferentes especies y la distribución de las mismas por clases de tamaño (Gadow et al., 2007).

Las especies arbóreas y arbustivas del matorral espinoso tamaulipeco presentan valores altos de riqueza específica $(S=32)$ y densidad $(>16,983$ ind/ha) respecto a otros matorrales del noreste de México (Heiseke y Foroughbakhch, 1985; Alanís et al., 2008; González et al., 2010; Jiménez et al., 2009). En este tipo de ecosistema la ganadería extensiva a gran escala ha sido practicada durante los últimos años (Foroughbakhch et al., 2009). Actualmente, las actividades productivas más comunes son la ganadería (intensiva y extensiva) y la agricultura de temporal (Jiménez et al., 2012). Con estas actividades se ha tenido como resultado la pérdida de la calidad y el número de especies forrajeras, seguida de una reducción de la capa vegetal que cubre y protege el suelo (González et al., 2004).

Recientemente se han realizado investigaciones que generan información fitosociológica $\mathrm{y}$ dasométrica de las especies arbóreas y arbustivas que se regeneran en el MET después de las principales 
perturbaciones, con el objetivo de evaluar cómo son las comunidades vegetales resultantes desde el punto de vista de su composición (Alanís et al., 2008; Jiménez et al., 2009; Jiménez \& Alanís, 2012; Jiménez et al., en prensa). Estas investigaciones son importantes para evaluar el estado post-perturbación de las áreas regeneradas, pero hace falta tener una comunidad de referencia para diseñar adecuadamente programas de restauración ecológica. El empleo de este tipo de información se ha incrementado entre los científicos, técnicos y gestores de sistemas naturales, ya que es el punto de partida para la correcta toma de decisiones en programas de restauración ecológica (Alanís et al., 2011; Jiménez et al., 2012).

Según la Sociedad de Restauración Ecológica (Society for Ecological Restoration, 2006), un ecosistema de referencia es un ecosistema verdadero o su modelo conceptual que se usa para establecer metas y planear un proyecto de restauración, y más adelante, para evaluarlo. El concepto de sistema de referencia debe representar un esquema de desarrollo avanzado que se encuentra en algún punto de la trayectoria ecológica deseada del ecosistema que se restaurará (Clewell et al., 2004).

La investigación tuvo como objetivo caracterizar el componente arbóreo y arbustivo $(\geq 5 \mathrm{~cm}$ de diámetro) de una comunidad de referencia en el matorral espinoso tamaulipeco, mediante: 1) Índice de riqueza de Margalef, 2) índice de diversidad de Shannon-Weaver) y 3) las variables estructurales de abundancia $\left(A R_{i}\right)$, dominancia (área de copa $\mathrm{o}$ cobertura) $\left(D R_{i}\right)$, frecuencia $\left(F R_{i}\right)$, así como el índice de valor de importancia (IVI) para cada especie.

\section{Materiales y métodos.}

La presente investigación se desarrolló en el Matorral-Escuela de la Facultad de Ciencias Forestales de la Universidad Autónoma de Nuevo León (Figura 1) en el municipio de Linares, Nuevo León (noreste de México). Las coordenadas de ubicación son $24^{\circ} 46^{\prime} 56.7^{\prime \prime}$ de latitud, y 99³1'34.3" de longitud, con una altitud de $366 \mathrm{msnm}$.

Para cumplir con el objetivo planteado, se seleccionó un área del MET que no tuviera registro histórico de ninguna actividad productiva que implicara la remoción total de la vegetación (matarrasa), agricultura o establecimiento de pastos para consumo del ganado, entre otras. El área seleccionada se encuentra delimitada por un cerco perimetral desde hace 28 años (1984-2012), donde se tiene la certeza que no se han desarrollado actividades que hayan causado algún tipo de perturbación. En el verano del año 2012 se establecieron cuatro sitios de muestreo distribuidos aleatoriamente. Las dimensiones de los sitios de muestreo fueron de $40 \times$ $40 \mathrm{~m}\left(1600 \mathrm{~m}^{2}\right)$.

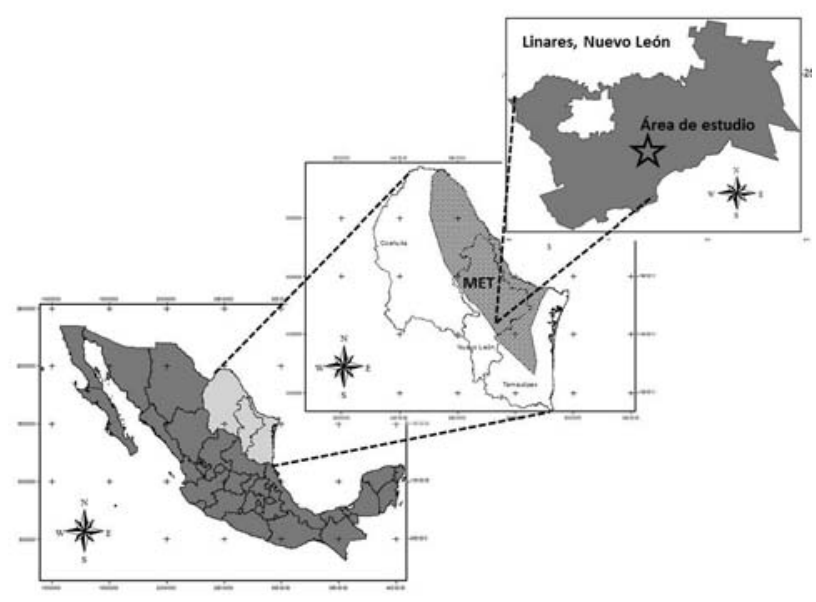

Figura 1. Localización de una comunidad de referencia de matorral espinoso tamaulipeco en el estado de Nuevo León, México.

En los sitios de muestro se realizó un censo de todas las especies arbóreas y arbustivas $\left(d_{0.10 \mathrm{~m}}>5\right.$ $\mathrm{cm})$. A cada individuo se le hicieron mediciones dasométricas de altura total $(h)$, diámetro $\left(d_{0.10} \mathrm{~m}\right) \mathrm{y}$ diámetro de copa. La medición del diámetro se efectuó a $0.10 \mathrm{~m}$ sobre el suelo, siendo una medida estándar empleada para especies arbóreas y arbustivas del MET (Gómez, 2000; Alanís et al., 2008; Jiménez y Alanís, 2012).

Para cada especie se determinó su abundancia, de acuerdo con el número de árboles, su cobertura, en función del área de copa, y su frecuencia con base en su presencia en los sitios de muestreo. Las variables relativizadas se utilizaron para obtener un valor ponderado a nivel de taxón denominado Índice de Valor de Importancia (IVI), que adquiere valores porcentuales en una escala de 0 a 100 (Mostacedo y Fredericksen, 2000).

Para evaluar la contribución estructural de las especies en la comunidad de estudio se utilizó la estimación de las siguientes variables estructurales: abundancia, cobertura, frecuencia e índice de valor de importancia. Las fórmulas para estos cálculos se describen a continuación.

$$
\begin{gathered}
A_{i}=N_{i} / E \\
A R_{i}=\left(A_{i} \sum_{i=1 \ldots n} A_{i}\right) \times 100
\end{gathered}
$$

donde $A_{i}$ es la abundancia absoluta, $A R_{i}$ es la abundancia relativa de la especie $i$ respecto a la abundancia total, $N_{i}$ es el número de individuos de la especie $i$, y $E$ la superficie de muestreo (ha).

La cobertura relativa se evaluó mediante:

$$
D_{i}=A b_{i} / E(h a)
$$




$$
D R_{i}=\left(D_{i} / \sum_{i=1 . . . n} D_{i}\right) \times 100
$$

dónde $D_{i}$ es la cobertura absoluta, $D R_{i}$ es cobertura relativa de la especie $i$ respecto a la cobertura, $A b$ el área de copa de la especie $i$ y $E$ la superficie (ha).

La frecuencia relativa se obtuvo con la siguiente ecuación:

$$
\begin{gathered}
F_{i}=P_{i} / N S \\
F R_{i}=\left(F_{i} / \sum_{i=1 \ldots n} F_{i}\right) \times 100
\end{gathered}
$$

dónde $F_{i}$ es la frecuencia absoluta, $F R_{i}$ es la frecuencia relativa de la especie $i$ respecto a la suma de las frecuencias, $P_{i}$ es el número de sitios en el que está presente la especie $i$ y NS el número total de sitios de muestreo. Cabe notar que como sólo se usaron cuatro parcelas de muestreo, la frecuencia tiene una baja capacidad de discriminación entre la especies respecto a esta variable estructural.

El índice de valor de importancia (IVI) se define como (Whittaker, 1972; Moreno, 2001):

$$
I V I=\frac{A R_{i}+D R_{i}+F R_{i}}{3}
$$

Para estimar la riqueza de especies se utilizó el índice de Margalef $\left(D_{M g}\right)$ y para la diversidad alfa el índice de Shannon-Weaver $\left(H^{\prime}\right)$ mediante las ecuaciones (Shannon, 1948; Magurran, 2004):

$$
\begin{gathered}
D_{M g}=\frac{(S-1)}{\ln (N)} \\
H^{\prime}=-\sum_{i=1}^{S} p_{i} \times \ln \left(p_{i}\right) \\
p_{i}=n_{i} / N
\end{gathered}
$$

Donde $S$ es el número de especies presentes, $N$ es el número total de individuos y $n_{i}$ es el número de individuos de la especie $i$.

\section{Resultados.}

Se registraron 21 especies arbóreas y arbustivas, distribuidas en 20 géneros y 14 familias (Tabla 1). La familia más rica en especies fue Fabaceae con siete especies (Parkinsonia texana, Prosopis levigaeta, Acacia amentacea, Acacia farnesiana, Ebenopsis ebano, Eysenhardtia polystachya y Havardia pallens), de las cuales Acacia amentacea fue la que presentó los valores mayores de abundancia relativa y cobertura relativa. Sin embargo, Diospyros texana fue la especie con mayor densidad relativa entre todas las especies presentes, con un valor mayor al reportado por Jiménez \& Alanís (2012), pero menor que el reportado por Canizales et al. (2009). Cabe mencionar que estas investigaciones tenían otros objetivos y analizaron a todos los individuos $>1 \mathrm{~cm}$ de diámetro, mientras que para el presente estudio solamente se registraron todos los individuos $>5 \mathrm{~cm}$ de diámetro, por lo que los resultados no son completamente comparables. Jiménez et al. (2012) registraron una cantidad menor de riqueza especies en un MET en el noreste de México con historial agrícola (Tabla 1).

La cobertura fue de $13,973 \mathrm{~m}^{2} \mathrm{ha}^{-1}$, lo que indica una cobertura superior al $100 \%$ y por lo tanto una considerable sobreposición de las copas. La especie que presentó mayor cobertura fue Acacia amentacea, con $7,635 \mathrm{~m}^{2} \mathrm{ha}^{-1}$, equivalente a $54.6 \%$ del total de la cobertura de la comunidad. Las especies que le siguieron fueron Diospyros texana y Cordia boissieri, con $14.9 \%$ y $9.2 \%$, respectivamente.

A nivel específico fue Acacia amentacea (IVI = $26.94 \%$ ) el taxón que presentó un IVI más elevado, mientras que la segunda y la tercera especie con mayor importancia fueron Diospyros texana, con $15.57 \%$, y Havardia pallens, con $13.01 \%$. (Tabla 2). Jiménez et al. (2009) reportaron a Bernardia myricaefolia y Eysenhardtia texana como las especies con mayor peso ecológico (IVI) en un MET con historial de ganadería extensiva; los mismos autores reportan en el mismo estudio a Diospyros texana y Acacia farnesiana con mayor peso ecológico (IVI) en un MET con historial de agricultura. El presente estudio difiere del de Jiménez et al. (2009), en el que se evaluó la misma comunidad, pero con diferentes tipos y niveles de perturbación (Tabla 2).

Los modelos paramétricos son modelos matemáticos que describen de forma gráfica la relación entre la abundancia y las especies ordenadas en categorías de la más a la menos abundante (Villareal et al. 2006). La distribución de diversidaddominancia del estudio corresponde al modelo paramétrico de series logarítmicas, donde se aprecia que hay un pequeño número de especies abundantes y una gran proporción de especies poco abundantes, lo que determina que las curvas sean como una jota invertida (Figura 2).

La Figura 3 muestra la densidad de individuos por hectárea de acuerdo con las clases diamétricas registradas en el estudio. Se observa una línea de tendencia exponencial negativa en la densidad de individuos conforme aumentan sus diámetros, siendo la clase $4-8 \mathrm{~cm}$ de diámetro la que presentó mayor número de individuos (1259 ind./ha). Esto indica que hay un gran número de individuos en las clases diamétricas menores, mostrando que el área se encuentra en un estado de regeneración activo. Caso contrario, hubo un pequeño número de individuos (19 ind./ha) con diámetros $>17 \mathrm{~cm}$. Esta información concuerda con Jiménez et al. (2012). 


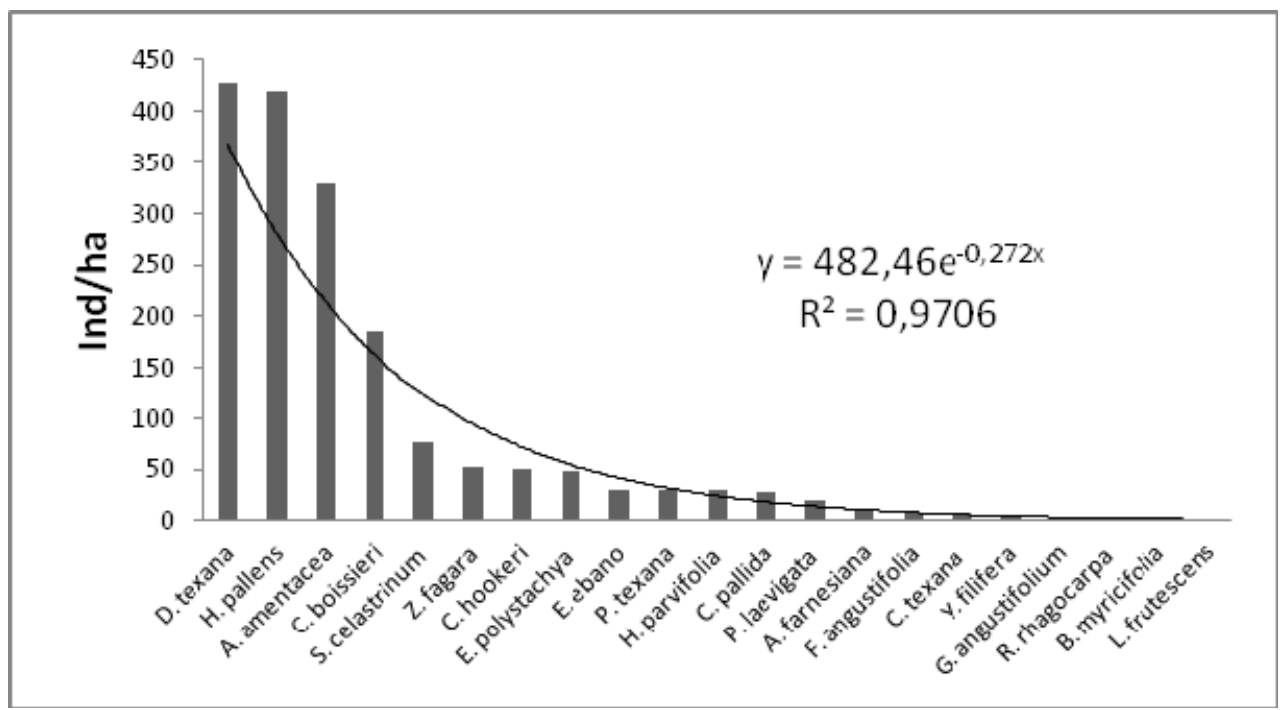

Figura 2. Modelo paramétrico de distribución dominancia-diversidad.

\section{Diversidad.}

La riqueza específica fue de 21 especies; el índice de Margalef $\left(D_{M g}\right)$ tuvo un valor de de 2.26 y el índice de Shannon-Weaver fue de 1.94. Estos valores superan a los reportados por Alanís et al. (2008), quienes registraron un valor inferior del índice de Margalef $\left(D_{M g}\right)$, y muy similar en diversidad $\left(H^{\prime}\right)$, al evaluar el MET con cuatro tipos de perturbación agropecuaria (matarrasa - remoción total de la vegetación-, ganadería intensiva, ganadería extensiva y agricultura). González et al. (2010), Jiménez y Alanís (2012) y Jiménez et al. (en prensa), quienes evaluaron áreas del MET con historial de uso productivo, reportan valores superiores a los presentados en este estudio. Una posible explicación de que los valores registrados en el estudio sean menores con respecto a los reportados en las investigaciones mencionadas, es que el ecosistema evaluado se encuentra en un estado más maduro, donde se encuentran ocupados los espacios y no existen condiciones para que se establezcan otras especies o nuevos individuos.

\section{Conclusiones.}

De acuerdo con los resultados obtenidos en la presente investigación, se destacan las siguientes conclusiones: 1) la comunidad estudiada presenta una riqueza específica y diversidad similar a comunidades con 20 años de regeneración post- silvoagropecuario, 2) presenta un estado de regeneración activo debido a la abundancia de individuos en las clases diamétricas menores, 3) la distribución de diversidad-dominancia

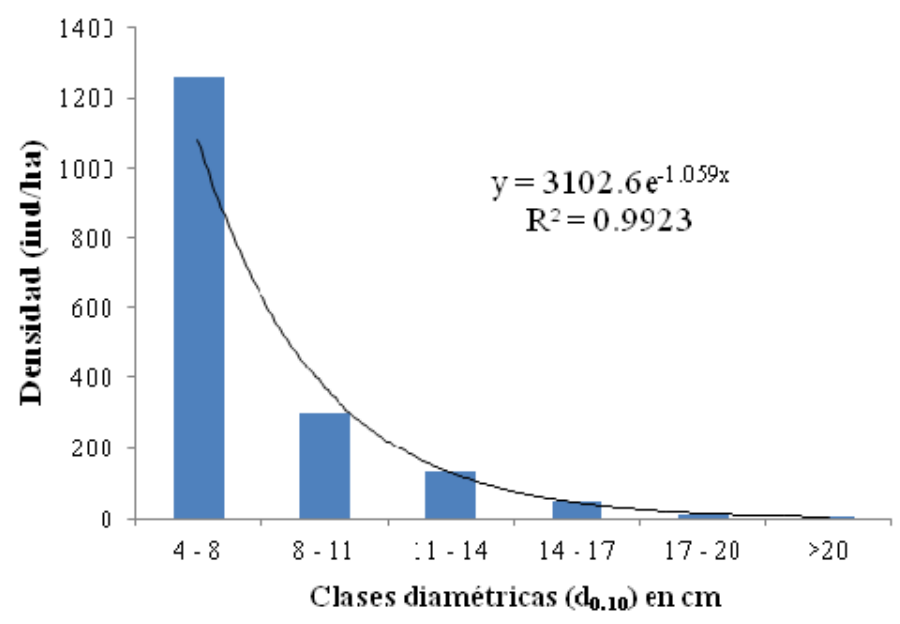

Figura 3. Distribución de frecuencias de las clases diamétricas en el área de estudio. 
muestra que existe un pequeño número de especies abundantes y una gran proporción de especies poco abundantes y 4) las especies con mayor importancia por su contribución estructural a la comunidad son Acacia amentacea, Diospyros texana y Havardia pallens. La investigación generó información cuantitativa de una comunidad madura de referencia del MET, la cual servirá como base para elaborar futuros programas de manejo, restauración y rehabilitación de este ecosistema.

\section{Agradecimientos.}

A la Facultad de Ciencias Forestales de la Universidad Autónoma de Nuevo León, por todas las facilidades otorgadas para el establecimiento y desarrollo de la investigación. Al equipo de trabajo del laboratorio de carbono de la Facultad de Ciencias Forestales y en especial a los compañeros José Ángel Sigala, Yazmin Hailen Ugalde, Román Ramírez y Alejandro Roblero, por su apoyo incondicional en el levantamiento de datos en campo. El proyecto fue financiado parcialmente por el proyecto PROMEP/103.5/12/3585.

\section{Literatura citada.}

Alanís E., Jiménez J., Aguirre O., Treviño E., Jurado E. \& González M. 2008. Efecto del uso del suelo en la fitodiversidad del matorral espinoso tamaulipeco. Ciencia UANL. 11(1):56-62.

Alanís E., Jiménez J., Valdecantos A., Pando M., Aguirre O. \& Treviño E.J. 2011. Caracterización de regeneración leñosa post-incendio de un ecosistema templado del Parque Ecológico Chipinque, México. Revista Chapingo serie Ciencias Forestales y del Ambiente. 17:31-39.

Canizales P. A., Alanís E., Aranda R., Mata J.M., Jiménez J., Alanís G., Uvalle J. I. \& Ruíz M.G. 2009. Caracterización estructural del matorral submontano de la Sierra Madre Oriental, Nuevo León. Revista Chapingo Serie Ciencias Forestales y del Ambiente.15 (2):115-120.

Clewell A., Aronson J. \& Winterhalder K. 2004. Principios de SER International sobre la restauración ecológica. Sociedad Internacional para la restauración ecológica. Tucson, Arizona, Estados Unidos de América. 6 pp.

Foroughbakhch R., Hernández P.J., Alvarado V.M., Céspedes C.E., Rocha E.A. \& Cárdenas M.L. 2009. Leaf biomass determination on woody shrub species in semiarid zones. Agroforestry Systems.77:181-192.

Gadow K.V., Sánchez O.S. \& Álvarez J.G. 2007. Estructura y Crecimiento del Bosque. Universidad de Göetingen, Alemania. $287 \mathrm{pp}$

Gómez A. 2000. Evaluación de áreas forestales de matorral utilizando un inventario multifásico. Tesis de Maestría. Facultad de Ciencias Forestales, Universidad Autónoma de Nuevo León, Linares, Nuevo León, México. 62 pp.
González H., Cantú I., Gómez M. \& Ramírez R. 2004. Plant water relations of thornscrub shrub species, northeasthern Mexico. Journal of Arid Environments. 58:483503.

González H., Ramírez R.G., Cantú I., Gómez M. \& Uvalle J.I. 2010. Composición y estructura de la vegetación en tres sitios del estado de Nuevo León, México. Polibotánica. 29:91-106.

Heiseke D. \& Foroughbakhch R. 1985. El matorral como recurso forestal. Reporte científico No. 1. Facultad de Silvicultura y Manejo de Recursos Renovables. Universidad Autónoma de Nuevo León. Linares, 31 pp.

Jiménez J., Alanís E., Aguirre O.A., Pando M. \& González M.A. 2009. Análisis sobre el efecto del uso del suelo en la diversidad estructural del matorral espinoso tamaulipeco. Maderas y Bosques. 15(3):5-20.

Jiménez J. \& Alanís E. 2012. Situación actual de los recursos forestales maderables en la región citrícola. Región Citrícola de Nuevo León: Su complejidad territorial en el marco global.

Jiménez J., Alanís E., González M.A., Aguirre O.A., Treviño E.J. \& Canizales P.A. En prensa. Characterizing woody species regeneration in areas with different land history tenure in the Tamaulipan Thornscrub, Mexico. The Southwestern Naturalist.

Jiménez J., Alanís E., Ruiz J.L., González M.A., Yerena J.I. \& Alanís G.J. 2012b. Diversidad de la regeneración leñosa del matorral espinoso tamaulipeco con historial agrícola en el NE de México. Ciencia UANL. 15(2):6671.

Magurran A.E. 2004. Measuring Biological Diversity. Blackwell Science. Oxford, UK. 256 pp.

Moreno C.E. 2001. Métodos para medir la biodiversidad. Manual y tesis SEA. Editado por Cooperación Iberoamericana (CYTED), Unesco (Orcyt) y SEA. Vol. 1. Pachuca, Hidalgo, México. 83 pp.

Mostacedo B. \& Fredericksen T.S. 2000. Manual de métodos básicos de muestreo y análisis en ecología vegetal. Editora El País, Santa Cruz, Bolivia. 87 pp.

Shannon C. 1948. The mathematical theory of communication. En: The mathematical theory of communication. Shannon C.E. \& Weaver W. (Ed). Univ. of Illinois Press Urbana. p. 29-125.

Society for Ecological Restoration (SER). 2006. International y la International Union for Conservation of Nature and Natural Resources - IUCN.

Villareal H., Álvarez M., Córdoba S., Escobar F., Fagua G., Gast F., Mendoza H., Ospina M. \& Umaña A.M. 2006.Métodos para el análisis de datos: una aplicación para resultados provenientes de caracterizaciones de biodiversidad: 191 (en) Manual de métodos para el desarrollo de inventarios de biodiversidad. Instituto de Investigación de Recursos Biológicos Alexander von Humboldt, Bogotá. Colombia.

Whittaker R.H. 1972. Evolution and measurement of species diversity. Taxon 21: 213-251. 
Tabla 1. Nombre científico, nombre común, familia y forma de crecimiento de las especies presentes en el área de estudio (ordenados por familia).

\begin{tabular}{llll}
\hline Nombre científico & Nombre común & Familia & $\begin{array}{l}\text { Forma de } \\
\text { crecimiento }\end{array}$ \\
\hline Acacia amentacea DC. & Gavia & Fabaceae & Arbustiva \\
Acacia farnesiana (L.) Willd. & Huizache & Fabaceae & Arbustiva \\
Ebenopsis ebano (Berland.) Barneby \& J.W. Grimes & Ébano & Fabaceae & Arbórea \\
Eysenhardtia polystachya (Ortega) Sarg. & Vara dulce & Fabaceae & Arbustiva \\
Havardia pallens (Benth.) Britton \& Rose & Tenaza & Fabaceae & Arbórea \\
Parkinsonia texana (A. Gray) S. Watson & Palo verde & Fabaceae & Arbórea \\
Prosopis laevigata (Humb. \& Bonpl. ex Willd.) M.C. Johnst. & Mezquite & Fabaceae & Arbórea \\
Helietta parvifolia (A. Gray) Benth. & Barreta & Rutaceae & Arbustiva \\
Zanthoxylum fagara (L.) Sarg. & Colima & Rutaceae & Arbustiva \\
Bernardia myricifolia (Scheele) Benth. \& Hook. f. & Oreja de ratón & Euphrobiaceae & Arbustiva \\
Castela texana (Torr. \& A. Gray) Rose & Chaparro amargoso & Simaroubaceae & Arbustiva \\
Celtis pallida Torr. & Granjeno & Cannabaceae & Arbustiva \\
Condalia hookeri M.C. Johnst. & Brasil & Rhamnaceae & Arbórea \\
Cordia boissieri A. DC. & Anacahuita & Boraginaceae & Arbórea \\
Diospyros texana Scheele & Chapote blanco & Ebenaceae & Arbórea \\
Forestiera angustifolia Torr. & Panalero & Oleaceae & Arbustiva \\
Leucophyllum frutescens (Berland.) I.M. Johnst. & Cenizo & Scrophulariaceae & Arbustiva \\
Guaiacum angustifolium Engelm. & Guayacan & Zygophyllaceae & Arbustiva \\
Randia rhagocarpa Standl. & Cruceto & Rubiaceae & Arbustiva \\
Sideroxylon celastrinum (Kunth) T.D. Penn. & Coma & Sapotaceae & Arbórea \\
Yucca filifera Chabaud & Yuca & Asparagaceae & Arbórea \\
\hline
\end{tabular}

Tabla 2. Densidad, cobertura, frecuencia e Índice de Valor de Importancia (IVI) de las especies presentes en una comunidad de referencia de matorral espinoso tamaulipeco (ordenados por familia).

\begin{tabular}{lcccccc}
\hline \multirow{2}{*}{ Nombre científico } & \multicolumn{2}{c}{ Abundancia } & \multicolumn{2}{c}{ Dominancia } & \multirow{2}{*}{ Frecuencia } & \multirow{2}{*}{ IVI } \\
\cline { 2 - 6 } (ind./ha) & rel & $\left(\mathrm{m}^{2} / \mathrm{ha}\right)$ & rel & & \\
\hline Acacia amentacea & 328 & 18.61 & 7635.72 & 54.65 & 7.55 & 26.94 \\
Acacia farnesiana & 9 & 0.53 & 60.21 & 0.43 & 3.77 & 1.58 \\
Ebenopsis ebano & 31 & 1.77 & 62.24 & 0.45 & 1.89 & 1.37 \\
Eysenhardtia polystachya & 48 & 2.75 & 223.61 & 1.60 & 7.55 & 3.96 \\
Havardia pallens & 419 & 23.75 & 1077.82 & 7.71 & 7.55 & 13.00 \\
Parkinsonia texana & 31 & 1.77 & 249.79 & 1.79 & 5.66 & 3.07 \\
Prosopis laevigata & 20 & 1.15 & 33.71 & 0.24 & 1.89 & 1.09 \\
Helietta parvifolia & 30 & 1.68 & 131.13 & 0.94 & 5.66 & 2.76 \\
Zanthoxylum fagara & 52 & 2.92 & 217.96 & 1.56 & 7.55 & 4.01 \\
Bernardia myricifolia & 2 & 0.09 & 11.79 & 0.08 & 1.89 & 0.69 \\
Castela texana & 5 & 0.27 & 3.69 & 0.03 & 1.89 & 0.73 \\
Celtis pallida & 28 & 1.60 & 76.34 & 0.55 & 3.77 & 1.97 \\
Condalia hookeri & 50 & 2.84 & 446.79 & 3.20 & 7.55 & 4.53 \\
Cordia boissieri & 186 & 10.55 & 1292.69 & 9.25 & 7.55 & 9.12 \\
Diospyros texana & 427 & 24.20 & 2088.56 & 14.95 & 7.55 & 15.56 \\
Forestiera angustifolia & 8 & 0.44 & 44.03 & 0.32 & 3.77 & 1.51 \\
Leucophyllum frutescens & 2 & 0.09 & 5.15 & 0.04 & 1.89 & 0.67 \\
Guaiacum angustifolium & 3 & 0.18 & 3.54 & 0.03 & 1.89 & 0.70 \\
Randia rhagocarpa & 3 & 0.18 & 3.89 & 0.03 & 1.89 & 0.70 \\
Sideroxylon celastrinum & 77 & 4.34 & 289.76 & 2.07 & 7.55 & 4.65 \\
Yucca filifera & 5 & 0.27 & 14.82 & 0.11 & 3.77 & 1.38 \\
& 1763 & 100 & 13973 & 100 & 100 & 100 \\
\hline
\end{tabular}

\footnotetext{
${ }^{1}$ Facultad de Ciencias Forestales; Universidad Autónoma de Nuevo León, Carretera Nacional km 145, Apartado Postal 41, Código Postal 67700, Linares, Nuevo León, México, biologomora@gmail.com.
} 